\title{
Distribution longitudinale des bryophytes d'un fleuve médi- térranéen du N.E. de l'Espagne : Le Fluvià.
}

\author{
Josep Peñuelas 1
}

Jordi Catalan'

\begin{abstract}
La végétation bryophytique du Fluvià, fleuve méditerranéen à bassin calcaire, a été étudié. 19 espèces aquat iques (submergées) et 27 espèces hygrophiles (entre 0 et $20 \mathrm{~cm}$ au dessus du niveau de l'eau) ont été trouvées. Les premières suivent une distribution zonale. qui a permis de diviser la rivière en trois secteurs : le cours supérieur, dominé par Barbula ehrenbergii, le cours moyen, dominé par Rhynchostegium riparioides et le cours inférieur, qui constitue le domaine des phanérogames aquatiques du genre Potamogeton. Les espèces hygrophyles, au contraire, se distribuent plus régulièrement tout au long de la rivière.
\end{abstract}

Longltudinal distribution of bryophytes in the Fluvià, a mediterranean river in the northeast of Spain.

The bryophyte vegetation was studied in the Fluvià, a mediterranean river in a calcareous area. 19 aquatic species (submerged) and 27 hygrophilic species (between 0 and $20 \mathrm{~cm}$ above the water surface) have been found. The aquatic species followed a zonal distribution that could be used to divide the river into three sections : the upper course, dominated by Barbula ehrenbergii, the middle course, dominated by Rhynchostegium riparoides, and the lower course which was dominated by phanerograms of the genus Potamogeton. In contrast, the hygrophilic species were distributed more regularly along the course of the river.

\section{1. - Introduction}

Ce travail est une nouvelle contribution à la connaissance de la végétation bryophytique des fleuves de l'Espagne et s'inscrit à la suite de celui de Penuelas (1983). Cet auteur citait divers travaux sur les mousses de fleuves et de torrents, notamment: Glime (1968) pour l'Amérique, Bonnard \& Michon (1981) pour la France, Empain (1973) pour la Belgique, Muhle et al (1979) pour l'Europe centrale, Wat. son (1919), Holmes \& Whitton (1977), Merry et al (1981) pour l'Angleterre, et, finalement, pour l'Espagne, Allorge (1947), Vigon (1977) et Gil \& Varó (1981). Les fleuves objets des études ici référencées coulent dans des régions dont le climat est soit atlantique soit continental humide. Dans le présent travail, les recherches ont été menées dans le Fluvià, fleuve typiquement méditérranéen. Notre but principal a été d'étudier la végétation bryophytique submergée et ses variations au long de l'année; on a

1. Departament d'Ecologia, Facultat de Biologia, Diagonal 645 , Barcelona, Espagne. cherché aussi à la comparer aux mousses hygrophiles qui poussent sur les rives, sous un régime d'humidité très élevée et soumises à des périodes d'immersion lorsque le débit augmente.

\section{2. - Caractéristiques du fleuve étudié}

Le Fluvià nait dans les pré-Pyrénées, à 1000 mètres d'altitude; il a une longueur de $97 \mathrm{~km}$ et la surface de son bassin peut être estimée à quelques $1100 \mathrm{~km}^{2}$. L'apport qu'il reçoit est de $225 \mathrm{Hm}^{3}$ en moyenne annuelle. Les terrains qu'il traverse sont calcaires et d'origine alluviale. Il présente une légère pollution (Tableau 1) dans son cours supérieur, dans la région de la Garrotxa ; cette pollution est due aux nombreux effluents urbains, industriels et agricoles qu'il reçoit à quelques kilomètres de sa source, dans les vallées d'en Bas et d'Olot. Dans cette région, le climat est humide avec plus de $1000 \mathrm{~mm}$ de précipitation annuelle. Plus tard, le fleuve pénètre dans les régions du Gironès et de l'Empordà, dont le climat est nettement méditerranéen $(600 \mathrm{~mm}$ de précipitation annuelle), et où il ne rencontre que peu de centres urbains ou industriels. 


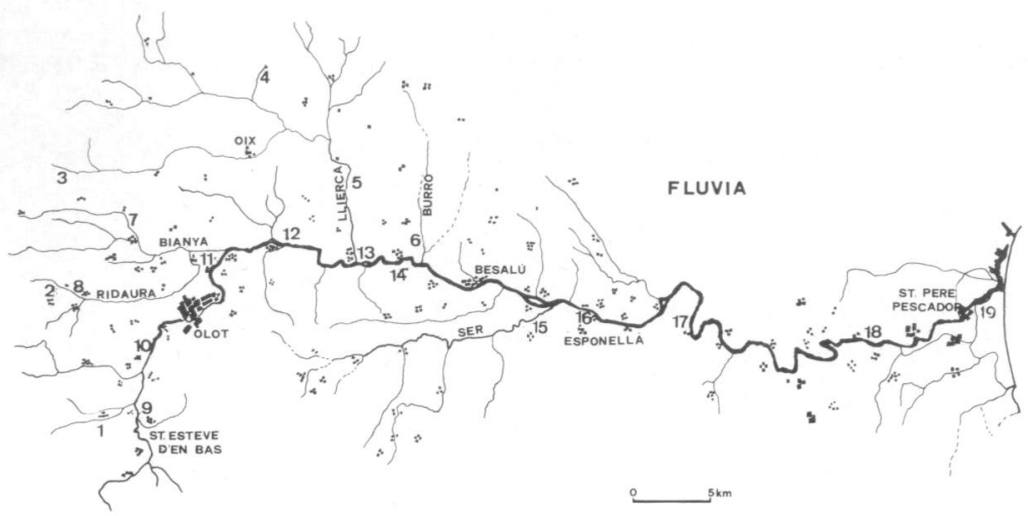

Fig. 1. Carte de la zone étudiée, avec la localisation des stations et des principales agglomérations urbaines.

TABleav I. Caractéristiques physico-chımiques de l'eau à Olot et Esponellà.

\begin{tabular}{|c|c|c|c|c|c|c|}
\hline & \multicolumn{3}{|c|}{ Olot } & \multicolumn{3}{|c|}{ Esponellà } \\
\hline & Moven & Maximum & Minimum & Moyen & Maximum & Minimum \\
\hline Debit $\mathrm{m}^{3} \mathrm{~s}$ & 1.8 & 7.9 & 0.1 & 6.7 & 28 & 1.3 \\
\hline $\mathrm{O}_{2} \mathrm{mg} / \mathrm{l}$ & 10 & 12 & 7 & 10 & 12 & 8 \\
\hline \multicolumn{7}{|l|}{ Matière particulaire } \\
\hline $\mathrm{mg} / \mathrm{l}$ & 6 & 26 & 2 & 8 & 16 & 4 \\
\hline Colifornes $/ 100 \mathrm{~cm}^{3}$ & 50000 & 90000 & 10000 & 2000 & 4000 & 100 \\
\hline $\mathrm{Cl}-\mathrm{mg} / \mathrm{l}$ & 29 & 38 & 19 & 35 & 44 & 28 \\
\hline $\mathrm{SO}_{4}=\mathrm{mg} / 1$ & 40 & 48 & 29 & 270 & 364 & 194 \\
\hline $\mathrm{SiO}_{3}=\mathrm{mg} / \mathrm{l}$ & 8.8 & 9.5 & 8 & 7.7 & 12 & 6 \\
\hline Alcalinité $\mathrm{mg} \mathrm{CaCO}_{3} \mathrm{l}$ & 185 & 256 & 25 & 187 & 230 & 66 \\
\hline $\mathrm{PO}_{4}^{3}-\mathrm{mg} / 1$ & 1.8 & 3.4 & 1.1 & 0.2 & 0.3 & 0.1 \\
\hline
\end{tabular}


Le régime du Fluvià est pluvial, avec une légère tendance nivo pluviale, à cause de l'origine prépy. rénéen de ses affluents de la rive gauche: Bianya, LLierca et Burrò.

\section{3. - Méthodologie}

Quatre campagnes d'échantillonnages ont été effectuées, en Novembre, Février, Mai et Août 1983. A chaque sortie, 19 stations étaient visitées (fig. 1). Les données physico-chimiques de l'eau pour Olot et pour Esponellà ont été fournies par le Commissariat des Eaux des Pyrénées Orientales (M.O.P., 1983). Pour les autres stations, ces données furent obtenues à l'aide d'un conductimètre YSI et d'un pHmètre Metrohm E488.

Dans chaque station, la végétation bryophytique submergée fut échantillonnée intensivement; pour chaque espèce fut notée son abondance relative, selon une échelle conventionnelle $+.1,2,3,4,5$.

Pour les espèces hygrophiles habitant les rives, entre 0 et $20 \mathrm{~cm}$ au dessus du niveau de l'eau, seules furent notées la présence (+) ou l'absence (.).

La nomenclature utilisée pour les mousses est celle de Casas (1981) et pour les hépatiques celle de Duell (1983).

\section{4. - Résultats et Discussion}

Les caractéristiques physico-chimiques (Tableau 1) montrent qu'il s'agit d'une rivière dont les eaux sont alcalines, avec une lègère pollution organique au début de son cours.

Les espèces trouvées complètement submergées sont récapitulées dans le tableau 2, selon un ordre décroissant d'abondance. L'absence de bryophytes aquatiques à la station 9 peut être sûrement attribuée à la pollution organique. Elles sont également absentes dans les stations 13,17,18, dans le cours inférieur de la rivière, sur des substrats de galets ou de sédiments, là ou le courant est faible. La station 10 où les rejets urbains sont déjà assez dilués par l'eau des divers affluents, nous surprend par sa végétation bryophytique exubérante et diversifiée, dominee par Fontinalis antipyretica. Cette espèce forme des grandes masses (fructifiees au mois de Février) qui recouvrent presque totalement le lit rocheux de la rivière, se trouvant à leur tour recouvertes de Sphaerotilus natans, bactérie indicatrice de matière organique. Plus tard, au printemps et pendant l'été, cette bactérie se trouve bien moins développée. Ce fait s'accorde bien avec les expériences de Peñuelas (1984a), selon lesquelles Fontinalis antipyretica se révélait comme l'une des mousses les plus résistantes à la contamination organique. Une autre espèce également résistante est $R$ hynchostegium riparioides. En effet, de la station 2 à la station 8 , séparées d'à peine $1 \mathrm{Km}$, mais recevant des effluents agricoles (élevage de bétail), la communauté à Barbula ehrenbergit caractéristique des têtes de fleuve calcaire, se voit remplacée par le communauté des secteurs moyen et inférieur, dominée par Rhynchostegium riparioides.

Très peu de changements stationnels ont été notés au cours de cette étude. On a pu remarquer la récu. pération progressive des stations qui furent dévastées par des inondations antérieures au début de nos campagnes : la station 7 qui ne présentait pas de bryophytes lors des premières visites, se trouvait colonisee vers la fin par des populations encore peu développées, tandis que le point 13 avait totalement récupéré sa végétation rivulaire.

D'une façon globale, à partir de la végétation bryophytique et en suivant les idées de certains limnologistes (Margalef 1983), on peut subdiviser la rivière en trois secteurs correspondant avec les trois secteurs traditionnels appellés cours supérieur, moyen et inférieur (fig. 2) :

1) Zone proche de la source du fleuve et des affluents, sur terrains calcaire avec des eaux propres, avant la traversée des centres urbains importants (stations $1,2,3,4,5,6$ ). La végétation bryophyt $i$ que est dominée par Barbula ehrenbergii, fréquem. ment accompagnée par Cratoneuron commutatum, Bryum pseudotriquetrum et Jungermannia atrovi. rens. Ces espèces sont communes dans les fleuves de l'Espagne méditerranéenne calcaire (Allorge 1947 ; Sanchez \& Gil 1982 ; Peñuelas 1983b) ; beaucoup d'entre elles et particulièrement Barbula ehrenbergii forment des travertins, à cause de la précipitation de $\mathrm{CaCO}_{3}$ sur les feuilles.

2) Zone moyenne, avec des eaux déjà plus polluées dans la traversée des centres urbains, et avec une pente moins forte (stations 8, 9, 10, 11, 12, 13, 14, 15, 16). L'espèce Rhynchostegium riparioides y domine, accompagnée par Cinclidolus spp., Fissidens spp., Fontinalis spp., Leptodictyum riparium et Hygroamblystegium tenax. Dans les fleuves et torrents moins alcalins, Rhynchostegium riparioides prend encore plus d'importance (Peñuelas 1983). 


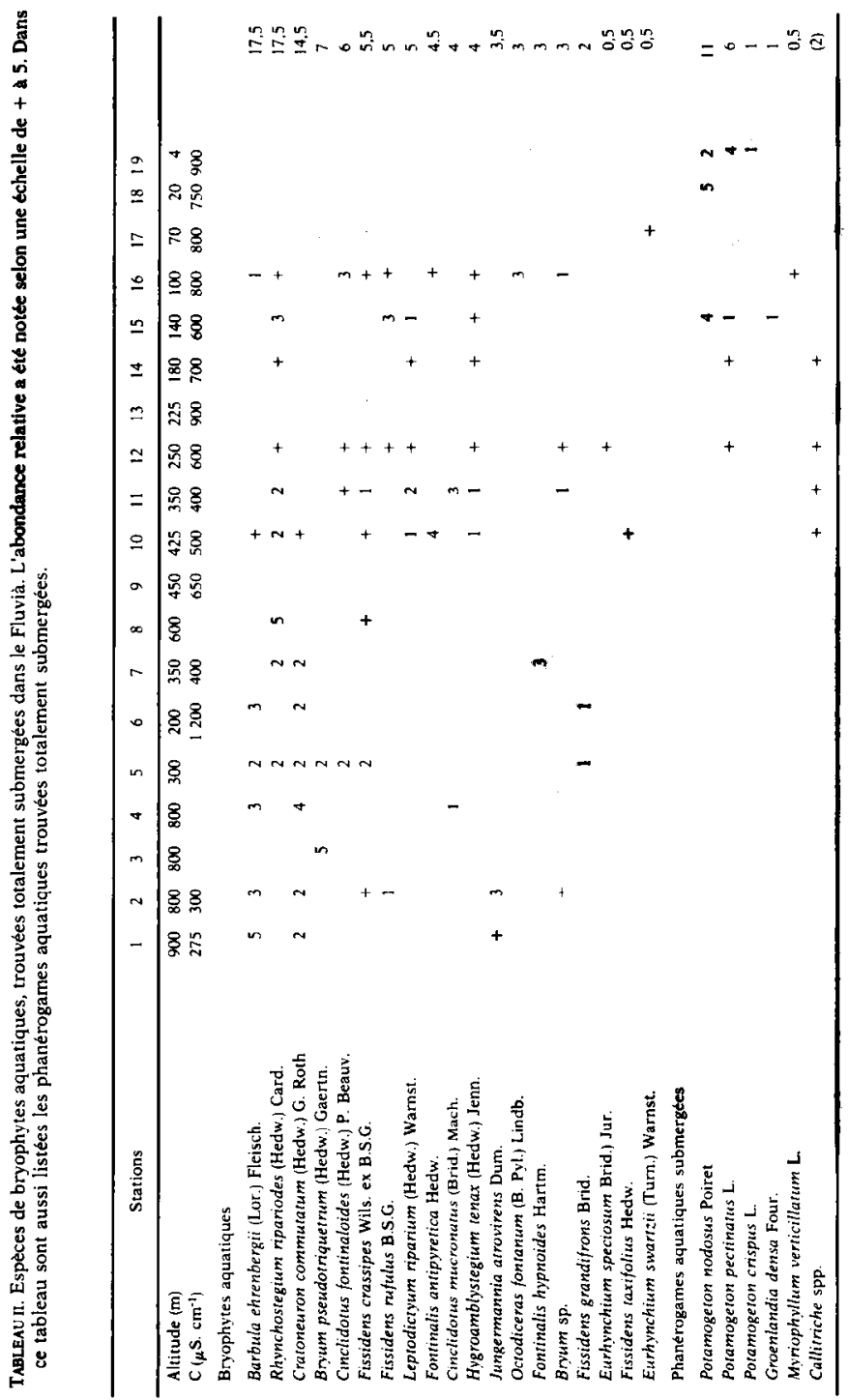


Cette espèce se trouve sur des roches et de gros cailloux, dans les zones généralement de faible profondeur et à courant fort. Cinclidotus fontinaloides domine dans les zones rocheuses soumises à des périodes d'émersion prolongées. Les espèces de Fissidens occupent les zones d'eau dormante et un peu plus profondes. Octodiceras fontanum, espèce très peu citée jusqu'à présent en Espagne (Periuelas 1984b) a été trouvée sur le mur du réservoir d'Esponellà (station 16), son habitat typique selon Wattez (1976).

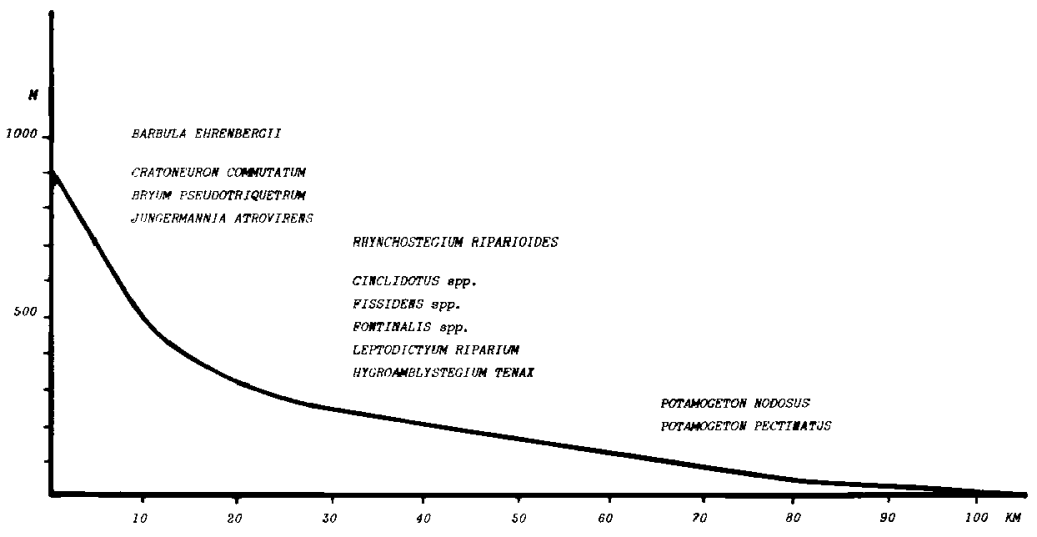

Fig 2. Profil longitudinal de la rivière, en signalant les communautés de macrophytes caractéristiques des

cours haut, moyen et bas. Les espèces sont ordonnees dans chaque groupe selon leur abondance relative.
3) Zone inférieure, avec une pente minimum et un courant très faible ; le substrat est vaseux. La végétation est dominée par des phanérogames, spécialement par les especes Potamogeton pectinatus et $P$. nodosus.

Cette distribution zonale ne semble pas être suivie par les espèces hygrophiles des rives (Tableau III) qui se trouvent plus ou moins tout le long du fleuve:Marchantia paleacea, Pellia endivitolia, Brachythecium rutabulum, Conocephalum conicum, Cratoneuron filicinum, Eurhynchium speciosum... 


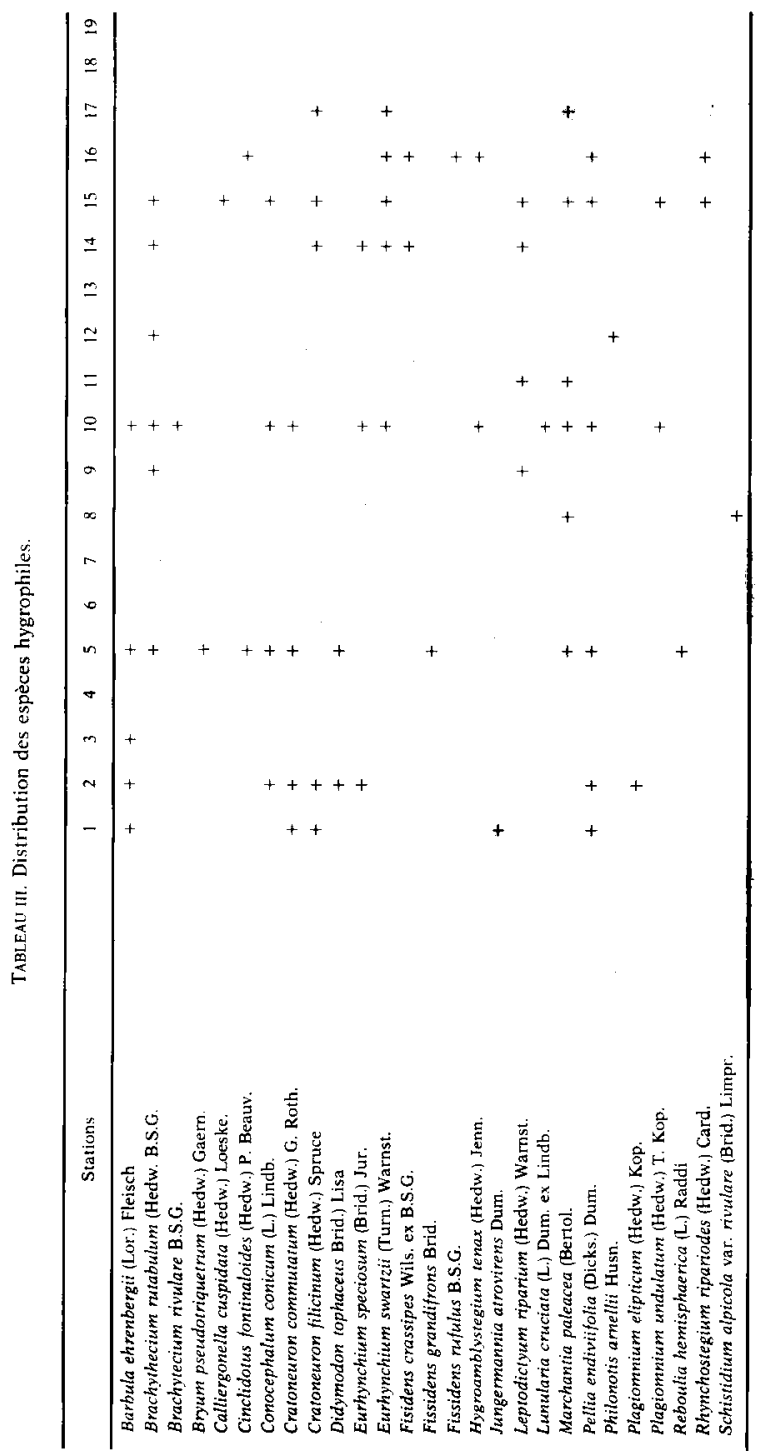




\section{Remerciements}

Les auteurs remercient vivement la Dra. Casas, qui les a aidé dans la determination des exemplaires délicats.

\section{Travaux cltés}

Allorge (P.). 1947,-Essai de Bryogeographie de la péninsule lbérque, Paul Lechevalier, Paris, 114 p.

Bonnard (R.) et Michon (A.). 198 1. - Les groupements de macrophy. tes aquatiques de la Loue. Annals Limnol., 17 (2): 105.120.

Casas (C.). 1981. - The mosses of Spain. An annotated check-list. Treballs de l"Instiut Balànic de Barcelona, $7: 1-57$.

Duell (R.). 1983.- Distribution of the european and macarronesian Liverworts (Hepticophytina). Bryologische Beisrage, 2 : $1-115$.

Empain (A.). 1973.- La végétation bryophitique aquatique et subaquatique de la Sambre belge ; son déterminisme écologique et ses relations avec des pollutions des eaux, Lejeunia, $69: 1.58$.

Gil (J.A.) et Varó (J.). 1981._- Estudio briosuciológico de las comunidades reófilas de Sierre Nevada (Espana). Criptogamie (Bryologie et Lichenologie, 2 (4); 423.440.

Glime (J.M.). 1968.- Ecological observations on some bryophytes in Apalachian mountain streams. Casfanea. 33 :300-325.

Holmes (N.T.) et Whitton (B.A.1. 1977. - Macrophytic vegetation of the river Swale, Yorkshire. Freshurat Biol. 7 : 545-558.

Margalef (R.). 1983 - Limnologia, Omega, Barcelona. $1010 \mathrm{p}$

Merry (D.G.), Slater (F.M.), et Randerson (P.F.). 1981.- The ripa- rian and aquatic vegetation of the river Wye, Joumal of Bio geography, $8: 313.327$.

Ministerio de Obras Publicas (M.O.P.). 1983.-Analisis de la calidad del agua, Publicaciones del M.O.P., Madrid.

Muhle (D.G.), Scherrer (M.) et Winkler (S.). 1979. - Wassermoose in den Nebenflüssen der Donau um Ulm. Mittleilungen des vereins fur Natumisenschaft und Mathematik Vim, 30 : $115-129$.

Peñuelas (j.). 1983 - Vegetación briofitica acuàtica del rio Muga y sus afluentes (Girona). Il Congreso de la Asociación Espanola de Limnologia, Murcia.

Penuelas (J.). 1984a. - Pigments of aquatic mosses of the river Muga (N.E. Spain) and their response to organic pollution. Lyndbergia. (sous presse).

Peñuelas (J.). 1984b. Octodiceras fontanum (B. Pyl.) Lindb a Catalunya. Folia Botanica Miscellanea, (sous press).

Sánchez (P.M.) et Gil (J.A.). 1982 - Vegetación criptogamica de las tobas de la provincia de Granada (Espana). Collectanea Botanica, 13 (1): 231.245.

Vigón (E.). 1977.-Estudio de la flora y vegetación muscinal acuàrica de la zona accidental asturiana. Tesis de la Universidad de Oviedo. Oviedo, $340 \mathrm{p}$.

Watson (W.). 1919.- The Bryophytes and Lichens of fresh water. J. Ecol, 7 (1): 71-83.

Wattez (J.R.). 1976. - Les bryophytes aquatiques et subaquatiques, bioindicateurs de la pollution des eaux douces. Pages 173-182. In Le pollation des eatu continentales, P. Pesson, Paris. 